\title{
Models and Algorithms for Tracking Target with Coordinated Turn Motion
}

\author{
Xianghui Yuan, Feng Lian, and Chongzhao Han \\ Ministry of Education Key Laboratory for Intelligent Networks and Network Security (MOE KLINNS), \\ School of Electronics and Information Engineering, Xian Jiaotong University, Xian 710049, China \\ Correspondence should be addressed to Feng Lian; lianfeng1981@mail.xjtu.edu.cn
}

Received 23 July 2013; Accepted 15 December 2013; Published 12 January 2014

Academic Editor: Shuli Sun

Copyright (C) 2014 Xianghui Yuan et al. This is an open access article distributed under the Creative Commons Attribution License, which permits unrestricted use, distribution, and reproduction in any medium, provided the original work is properly cited.

\begin{abstract}
Tracking target with coordinated turn (CT) motion is highly dependent on the models and algorithms. First, the widely used models are compared in this paper-coordinated turn (CT) model with known turn rate, augmented coordinated turn (ACT) model with Cartesian velocity, ACT model with polar velocity, CT model using a kinematic constraint, and maneuver centered circular motion model. Then, in the single model tracking framework, the tracking algorithms for the last four models are compared and the suggestions on the choice of models for different practical target tracking problems are given. Finally, in the multiple models (MM) framework, the algorithm based on expectation maximization (EM) algorithm is derived, including both the batch form and the recursive form. Compared with the widely used interacting multiple model (IMM) algorithm, the EM algorithm shows its effectiveness.
\end{abstract}

\section{Introduction}

The problem of tracking a single target with coordinated turn (CT) motion is considered. The motion of a civil aircraft can usually be modeled as moving by constant speed in straight lines and circle segments. The former is known as constant velocity $(\mathrm{CV})$ model and the latter is coordinated turn model. In tracking applications, only the position part of the state can be measured by the sensor and the turn rate $\omega$ is often unknown. So the measurement data can be seen as the incomplete data. This is a resource-constrained problem for tracking target with coordinated turn motion.

CT model is highly dependent on the choice of state components [1]. The turn rate $\omega$ can be augmented in the CT model, called ACT model. There are two types of ACT models: ACT model with Cartesian velocity and ACT model with polar velocity. The state vectors are $[x, y, \dot{x}, \dot{y}, \omega]^{\prime}$ and $[x, y, v, \phi, \omega]^{\prime}$, respectively. The two are both nonlinear models and have been compared in [2,3] based on EKF. For unscented Kalman filter (UKF) is a very efficient tool for nonlinear estimation $[4,5]$, here the two models are compared based on UKF.

When the target with CT motion has a constant speed, it satisfies a kinematic constraint: $V \cdot A=0$, where $V$ is the target velocity vector and $A$ is the target acceleration vector. If the dynamic model incorporates the constraint directly, it will become a highly nonlinear one. To avoid this nonlinearity, the kinematic constraint was incorporated into a pseudomeasurement model [6-8].

A maneuver-centered model is introduced in [9]. The state components are $[r, \theta, \omega]^{\prime}$. The model's state equation has a linear form, but its measurement equation is pseudolinear because the noise covariance is actually state dependent [10]. The center of the turn should be accurately determined, which is inherently a nonlinear problem.

Target dynamic models and tracking algorithms have intimate ties [1]. In the single model tracking framework, the tracking algorithms are interpreted and compared.

The interacting multiple model (IMM) approach has been generally considered to be the mainstream approach to maneuvering target tracking. It utilizes a bank of $N$ Kalman filters, each designed to model a different maneuver [11]. IMM algorithm is a suboptimal algorithm based on the minimum mean square error (MMSE) criterion. Under the MMSE criterion, to get the optimal estimation of the target state, the computational load grows exponentially when the measurements are increasing. In recent years, tracking target based on maximum a posteriori (MAP) criterion has received a lot of 
interest [12-17]. Expectation maximization (EM) algorithm is the state estimation approach based on MAP criterion. Us ing EM algorithm, the computational load grows linearly during per iteration and the optimal estimation based on MAP criterion can be achieved finally.

The existing EM algorithm to track maneuvering target can be classified into two categories: one formulates the maneuver as the unknown input [12-14] and the other formulates the maneuver as the system's process noise [15]. Aiming at the problem to track a target with CT maneuver, an EM algorithm is presented. The maneuver is formulated by the turn rate. First, the turn rate sequence is estimated using the EM algorithm. Then, with the estimated turn rate sequence, the target state sequence is estimated accurately.

The rest of this paper is organized as follows. Section 2 presents all the CT models' state equations and measurement equations. The tracking algorithms based on single model are interpreted in Section 3; the simulations are also presented. In Section 4, the batch and recursive EM algorithms are derived and compared with the IMM algorithm in simulation. Section 5 provides the paper's conclusions.

\section{Dynamic Models for CT Motion}

A maneuvering target can be modeled by

$$
\begin{gathered}
X_{k+1}=f_{k}\left(X_{k}\right)+w_{k}, \\
z_{k}=h_{k}\left(X_{k}\right)+e_{k},
\end{gathered}
$$

where $X_{k}$ and $z_{k}$ are target state and observation, respectively, at discrete time $t_{k} ; w_{k}$ and $e_{k}$ are process noise and measurement noise sequences, respectively; $f_{k}$ and $h_{k}$ are vector-valued functions.

2.1. CT Model with Known Turn Rate. The coordinated turn motion can be described by the following equation:

$$
X_{k+1}=F\left(\omega_{k}\right) X_{k}+w_{k} .
$$

The measurement equation is:

$$
z_{k}=H_{\mathrm{CT}} X_{k}+e_{k}
$$

The components of state are $X=\left[\begin{array}{cccc}x & \dot{x} & y & \dot{y}\end{array}\right]^{\prime} . \omega_{k}$ stands for the turn rate in time $k$.

Where

$$
\begin{gathered}
F\left(\omega_{k}\right)=\left[\begin{array}{cccc}
1 & \frac{\sin \left(\omega_{k} T\right)}{\omega_{k}} & 0 & -\frac{1-\cos \left(\omega_{k} T\right)}{\omega_{k}} \\
0 & \cos \left(\omega_{k} T\right) & 0 & -\sin \left(\omega_{k} T\right) \\
0 & \frac{1-\cos \left(\omega_{k} T\right)}{\omega_{k}} & 1 & \frac{\sin \left(\omega_{k} T\right)}{\omega_{k}} \\
0 & \sin \left(\omega_{k} T\right) & 0 & \cos \left(\omega_{k} T\right)
\end{array}\right] \\
E\left[w_{k}\right]=0, \quad E\left[\begin{array}{ll}
w_{x} & \left.w_{y}\right]^{\prime}
\end{array}\right. \\
E\left[\begin{array}{l}
w_{k} w_{l}^{\prime}
\end{array}\right]=Q_{\mathrm{CT}} \delta_{k l} .
\end{gathered}
$$

Assume only position could be measured, where

$$
\begin{gathered}
H_{\mathrm{CT}}=\left[\begin{array}{llll}
1 & 0 & 0 & 0 \\
0 & 0 & 1 & 0
\end{array}\right] \\
E\left[e_{k}\right]=0, \quad E\left[e_{k} e_{l}^{\prime}\right]=R \delta_{k l} .
\end{gathered}
$$

This model assumes that the turn rate is known or could be estimated. When the range rate measurements are available, the turn rate could be estimated by using range rate measurements $[18,19]$. The tracking performance will be deteriorated when the assumed turn rate is far away from the true one. This model is usually used as one of the models in a multiple models framework.

2.2. ACT Model with Cartesian Velocity. In this model, the state vector is chosen to be $X=[x, y, \dot{x}, \dot{y}, \omega]^{\prime}$; the state space equation can be written as

$$
X_{k+1}=f_{\mathrm{ACT} 1}\left(X_{k}\right)+G_{\mathrm{ACT} 1} w_{k}
$$

where

$$
\begin{aligned}
& f_{\mathrm{ACT} 1}(X)=\left[\begin{array}{c}
x+\frac{\dot{x}}{\omega} \sin (\omega T)-\frac{\dot{y}}{\omega}(1-\cos (\omega T)) \\
y+\frac{\dot{x}}{\omega}(1-\cos (\omega T))+\frac{\dot{y}}{\omega}(\sin (\omega T)) \\
\dot{x} \cos (\omega T)-\dot{y} \sin (\omega T) \\
\dot{x} \sin (\omega T)+\dot{y} \cos (\omega T) \\
\omega
\end{array}\right] \text {, } \\
& G_{\mathrm{ACT} 1}=\left[\begin{array}{ccccc}
\frac{T^{2}}{2} & 0 & T & 0 & 0 \\
0 & \frac{T^{2}}{2} & 0 & T & 0 \\
0 & 0 & 0 & 0 & 1
\end{array}\right]^{\prime}, \\
& w=\left[\begin{array}{lll}
w_{x} & w_{y} & w_{\omega}
\end{array}\right]^{\prime}, \\
& E\left[w_{k}\right]=0, \quad E\left[w_{k} w_{l}^{\prime}\right]=Q_{\mathrm{ACT} 1} \delta_{k l} .
\end{aligned}
$$

Assume only position could be measured, the measurement equation can be written as

$$
z_{k}=H_{\mathrm{ACT} 1} X_{k}+e_{k}
$$

where

$$
\begin{gathered}
H_{\mathrm{ACT} 1}=\left[\begin{array}{lllll}
1 & 0 & 0 & 0 & 0 \\
0 & 1 & 0 & 0 & 0
\end{array}\right], \\
E\left[e_{k}\right]=0, \quad E\left[e_{k} e_{l}^{\prime}\right]=R \delta_{k l} .
\end{gathered}
$$

2.3. ACT Model with Polar Velocity. This model's state vector is $X=[x, y, v, \phi, \omega]^{\prime}$, and the dynamic state equation is given by

$$
X_{k+1}=f_{\mathrm{ACT} 2}\left(X_{k}\right)+G_{\mathrm{ACT} 2} w_{k}
$$


where

$$
\begin{aligned}
& f_{\mathrm{ACT} 2}(X)=\left[\begin{array}{c}
x+\left(\frac{2 v}{\omega}\right) \sin \left(\frac{\omega T}{2}\right) \cos \left(\phi+\frac{\omega T}{2}\right) \\
y+\left(\frac{2 v}{\omega}\right) \sin \left(\frac{\omega T}{2}\right) \sin \left(\phi+\frac{\omega T}{2}\right) \\
v \\
\phi+\omega T \\
\omega
\end{array}\right] \\
& G_{\mathrm{ACT} 2}=\left[\begin{array}{ccccc}
0 & 0 & T^{2} & 0 & 0 \\
0 & 0 & 0 & \frac{T^{2}}{2} & T^{2}
\end{array}\right]^{\prime} \\
& w=\left[\begin{array}{ll}
w_{v} & w_{\omega}
\end{array}\right]^{\prime} \\
& E\left[w_{k}\right]=0, \quad E\left[w_{k} w_{l}^{\prime}\right]=Q_{\mathrm{ACT} 2} \delta_{k l} .
\end{aligned}
$$

However the measurement equation is the same as (11) to (13).

2.4. Kinematic Constraint Model. For a constant speed target, the acceleration vector is orthogonal to the velocity vector:

$$
C(X)=V \cdot A=0,
$$

where $V$ is the target velocity vector and $A$ is the target acceleration vector.

This kinematic constraint can be used as a pseudomeasurement. The state vector is chosen to be $X=$ $\left[\begin{array}{llllll}x & \dot{x} & \ddot{x} & y & \dot{y} & \ddot{y}\end{array}\right]^{\prime}$. So the dynamic model is the constant acceleration (CA) model, given by

$$
X_{k+1}=F X_{k}+G w_{k}
$$

where

$$
\begin{aligned}
& F=\left[\begin{array}{cccccc}
1 & T & \frac{T^{2}}{2} & 0 & 0 & 0 \\
0 & 1 & T & 0 & 0 & 0 \\
0 & 0 & 1 & 0 & 0 & 0 \\
0 & 0 & 0 & 1 & T & \frac{T^{2}}{2} \\
0 & 0 & 0 & 0 & 1 & T \\
0 & 0 & 0 & 0 & 0 & 1
\end{array}\right] \\
& G=\left[\begin{array}{cccccc}
\frac{T^{2}}{2} & T & 1 & 0 & 0 & 0 \\
0 & 0 & 0 & \frac{T^{2}}{2} & T & 1
\end{array}\right]^{\prime} \\
& w=\left[\begin{array}{ll}
w_{x} & w_{y}
\end{array}\right]^{\prime} \\
& E\left[w_{k}\right]=0, \quad E\left[w_{k} w_{l}^{\prime}\right]=Q_{\mathrm{CA}} \delta_{k l} .
\end{aligned}
$$

The measurement equation is given by

$$
z_{k}=H x_{k}+e_{k} \text {, }
$$

where

$$
\begin{gathered}
H=\left[\begin{array}{llllll}
1 & 0 & 0 & 0 & 0 & 0 \\
0 & 0 & 0 & 1 & 0 & 0
\end{array}\right] \\
E\left[e_{k}\right]=0, \quad E\left[e_{k} e_{l}^{\prime}\right]=R \delta_{k l} .
\end{gathered}
$$

The pseudomeasurement is

$$
\frac{V_{k \mid k}}{S_{k \mid k}} \cdot A_{k}+\mu_{k}=0,
$$

where $V_{k \mid k}=\left[\begin{array}{ll}\dot{x}_{k \mid k} & \dot{y}_{k \mid k}\end{array}\right]^{\prime}$ and $A_{k}=\left[\begin{array}{ll}\ddot{x}_{k} & \ddot{y}_{k}\end{array}\right]^{\prime}$.

$S_{k \mid k}$ is the filtered speed at time $k$ :

$$
\begin{gathered}
S_{k \mid k}=\sqrt{\dot{x}_{k \mid k}^{2}+\dot{y}_{k \mid k}^{2}}, \\
\mu_{k} \sim N\left(0, R_{k}^{\mu}\right), \\
R_{k}^{\mu}=r_{1}(\delta)^{k}+r_{0}, \quad 0 \leq \delta<1,
\end{gathered}
$$

where $r_{1}$ is chosen to be large for initialization and $r_{0}$ is chosen for steady-state conditions.

2.5. Maneuver-Centered CT Model. This model's state vector is given by $X=\left[\begin{array}{lll}r & \theta & \omega\end{array}\right]^{\prime}$. The process state space equation is

$$
X_{k+1}=\Phi X_{k}+\Gamma w_{k}
$$

where

$$
\begin{gathered}
\Phi=\left[\begin{array}{lll}
1 & 0 & 0 \\
0 & 1 & T \\
0 & 0 & 1
\end{array}\right] \\
\Gamma=\left[\begin{array}{ll}
1 & 0 \\
0 & \frac{T}{2} \\
0 & 1
\end{array}\right] \\
w=\left[\begin{array}{ll}
w_{r} & w_{\omega}
\end{array}\right]^{\prime}
\end{gathered}
$$$$
E\left[w_{k}\right]=0, \quad E\left[w_{k} w_{l}^{\prime}\right]=Q_{m} \delta_{k l} .
$$

Assume the center of the CT motion is $\left(\widehat{x}_{c}, \widehat{y}_{c}\right)$. The transformation between Cartesian coordinates and maneuvercentered coordinates is given by

$$
\begin{gathered}
r=\sqrt{\left(x-\hat{x}_{c}\right)^{2}+\left(y-\hat{y}_{c}\right)^{2}} \\
\theta=\tan ^{-1}\left(\frac{y-\hat{y}_{c}}{x-\hat{x}_{c}}\right) .
\end{gathered}
$$

So the measurement equation is given by

$$
z_{k}=H^{m} X_{k}+e_{k}
$$

where

$$
H^{m}=\left[\begin{array}{lll}
1 & 0 & 0 \\
0 & 1 & 0
\end{array}\right]
$$

$$
E\left[e_{k} e_{l}^{\prime}\right]=R^{m}=J_{r \theta} R J_{r \theta}^{\prime} .
$$

$J_{r \theta}$ is the Jacobian matrix based on (27), which leads to

$$
J_{r \theta}=\left[\begin{array}{cc}
\cos \theta & \sin \theta \\
-\sin \frac{\theta}{r} & \cos \frac{\theta}{r}
\end{array}\right] .
$$




\section{Tracking Algorithms in a Single Model Framework}

3.1. UKF Filter with ACT Models. If the turn rate is augmented to the state vector, it will become a nonlinear problem. The extended Kalman filter (EKF) has been used to track this kind of motion. Since unscented Kalman filter (UKF) is very suitable for nonlinear estimation $[4,5]$, here the UKF algorithm is introduced.

(i) Calculate the Weights of Sigma Points

$$
\begin{aligned}
W_{0}^{m} & =\frac{\lambda}{(n+\lambda)} W_{0}^{c} \\
& =\frac{\lambda}{(n+\lambda)}+\left(1-\alpha^{2}+\beta\right) W_{i}^{m} \\
& =W_{i}^{c}=\frac{0.5}{(n+\lambda)}, \quad i=1,2, \ldots 2 n,
\end{aligned}
$$

where $n$ is the dimension of the state vector. $\lambda=$ $\alpha^{2}(n+\kappa)-n$ is a scaling parameter. $\alpha$ determines the sigma points around $\bar{x}$ and is usually set to a small positive value (e.g., $1 e-3) . \kappa$ is a secondary scaling parameter which is usually set to 0 , and $\beta=2$ is optimal for Gauss distributions. Where the $\left(\sqrt{(n+\lambda) P_{x}}\right)_{i}$ is the $i$ th row of the matrix square root.

(ii) Calculate the Sigma Points

$$
\begin{gathered}
\xi_{k-1 \mid k-1}^{0}=\widehat{X}_{k-1 \mid k-1} \\
\xi_{k-1 \mid k-1}^{(i)}=\widehat{X}_{k-1 \mid k-1}+\left(\sqrt{(n+\lambda) P_{x}}\right)_{i} i=1,2, \ldots, n \\
\xi_{k-1 \mid k-1}^{(i)}=\widehat{X}_{k-1 \mid k-1}-\left(\sqrt{(n+\lambda) P_{x}}\right)_{i} \\
i=n+1, n+2, \ldots, 2 n .
\end{gathered}
$$

(iii) Time Update

$$
\begin{aligned}
\xi_{k}^{(i)} & =f_{k}\left(\xi_{k-1 \mid k-1}^{(i)}\right), \quad i=0,1, \ldots 2 n \widehat{X}_{k \mid k-1} \\
& =\sum_{i=0}^{2 n} W_{i}^{m} \xi_{k}^{(i)} P_{k \mid k-1} \\
& =\sum_{i=0}^{2 n} W_{i}^{c}\left(\xi_{k}^{(i)}-\widehat{X}_{k \mid k-1}\right)\left(\xi_{k}^{(i)}-\widehat{X}_{k \mid k-1}\right)^{\prime}+G Q_{k-1} G^{\prime} .
\end{aligned}
$$

(iv) Measurement Update. Because we assume the measurement equation is linear, the following is just the same as the traditional Kalman filter:

$$
\begin{aligned}
\widehat{z}_{k \mid k-1} & =H \widehat{X}_{k \mid k-1} S_{k} \\
& =H P_{k \mid k-1} H^{\prime}+R_{k} K_{k}
\end{aligned}
$$

$$
\begin{aligned}
& =P_{k \mid k-1} H^{\prime} S_{k}^{-1} \widehat{X}_{k \mid k} \\
& =\widehat{X}_{k \mid k-1}+K_{k}\left(z_{k}-z_{k \mid k-1}\right) P_{k \mid k} \\
& =P_{k \mid k-1}-K_{k} S_{k} K_{k}^{\prime} .
\end{aligned}
$$

For the cases where the measurement equation is also nonlinear, the measurement update can be referred to [10] for details.

3.2. Kinematic Constraint Tracking Filter. The Kalman filtering equations for processing this kinematic constraint as a pseudomeasurement are given below, where the filtered state estimate and error covariance after the constraint have been applied are denoted by $X_{k \mid k}^{C}$ and $P_{k \mid k}^{C}$, respectively [8].

(i) Time Update

$$
\begin{gathered}
\widehat{X}_{k \mid k-1}=F \widehat{X}_{k-1 \mid k-1}^{C} \\
P_{k \mid k-1}=F P_{k-1 \mid k-1}^{C} F^{\prime}+G Q_{k-1} G^{\prime} .
\end{gathered}
$$

(ii) Measurement Update. The measurement update is the same as (34).

(iii) Constraint Update

$$
\begin{aligned}
K_{k}^{C} & =P_{k \mid k} C_{k}^{T}\left[C_{k} P_{k \mid k} C_{k}^{\prime}+R_{k}^{\mu}\right]^{-1} \widehat{X}_{k \mid k}^{C} \\
& =\left[I-K_{k}^{C} C_{k}\right] \widehat{X}_{k \mid k} P_{k \mid k}^{C} \\
& =\left[I-K_{k}^{C} C_{k}\right] P_{k \mid k},
\end{aligned}
$$

where

$$
C_{k}=\frac{1}{S_{k \mid k}}\left[\begin{array}{llllll}
0 & 0 & \hat{\dot{x}}_{k \mid k} & 0 & 0 & \widehat{\dot{y}}_{k \mid k}
\end{array}\right] .
$$

\subsection{Maneuver-Centered Tracking Filter}

(i) Estimating Center of Maneuver. The center of the maneuver should be estimated from the measurements. It can be estimated through least square method which requires an iterative search procedure. The following simple geometrically oriented procedure of estimating the center was proposed in [9]. The main idea is as follows: if two points are on a circle then the perpendicular bisector of the chord between those points will pass through the center of the circle. The slope $(m)$ and $y$ intercept $(b)$ of the perpendicular bisector is given by

$$
\begin{gathered}
m=\frac{\left(x_{1}-x_{2}\right)}{\left(y_{2}-y_{1}\right)} \\
b=\frac{\left(y_{1}+y_{2}\right)}{2}-m \frac{\left(x_{1}+x_{2}\right)}{2},
\end{gathered}
$$


where $\left(x_{1}, y_{1}\right)$ and $\left(x_{2}, y_{2}\right)$ are the coordinates of the two points. The center can be given by

$$
\begin{gathered}
\widehat{x}_{c}=\frac{\left(b_{1}-b_{2}\right)}{\left(m_{2}-m_{1}\right)} \\
\widehat{y}_{c}=\frac{\left(m_{1} b_{2}-m_{2} b_{1}\right)}{\left(m_{1}-m_{2}\right)} .
\end{gathered}
$$

(ii) Maneuver Detection. In the absence of a maneuver, the target is assumed to be traveling in a straight line and modeled by a constant velocity $(\mathrm{CV})$ motion. (CV model is very simple and commonly used, which will not be listed here.) When the maneuver is detected, the filter switches to the maneuvercenter CT model. While the end of a maneuver is detected, the filter will then switch back to CV model.

Here a fading memory average of the innovations is used to detect if a maneuver occurs. The equation is given by

$$
u_{k}=\rho u_{k-1}+d_{k}
$$

with

$$
d_{k}=v_{k}^{\prime} S_{k}^{-1} v_{k},
$$

where $0<\rho<1, v_{k}$ is the innovation vector, and $S_{k}$ is its covariance matrix.

$u_{k}$ will have a chi-squared distribution with degrees

$$
n_{u}=n_{z} \frac{1+\rho}{1-\rho},
$$

where $n_{z}$ is the dimension of the measurement vector. When $u_{k}$ exceeds a threshold (e.g., $95 \%$ or $99 \%$ confidence interval), then a maneuver onset is declared. The end time of a maneuver will be determined in a similar fashion. The procedure can be referred to [9] for details.

\subsection{Simulation Results}

(i) The Scenario. The scenario simulated here is very similar to that described in [20]. It includes few rectilinear stages and few CT maneuvers. Four consecutive $180^{\circ}$ turns with rates $\omega=1.87,-2.8,5.6,-4.68$ are simulated, respectively, for scans $[56,150],[182,245],[285,314]$, and $[343,379]$. The target trajectory can be seen in Figure 1.

The initial target position and velocities are $X_{0}=60 \mathrm{~km}$, $Y_{0}=40 \mathrm{~km}, \dot{X}_{0}=-172 \mathrm{~km}$, and $\dot{Y}_{0}=246 \mathrm{~km}$. It is assumed that the sensor measures Cartesian coordinates $X$ and $Y$ directly. It is also assumed that $\sigma_{X}=\sigma_{Y}=100 \mathrm{~m}$ and the sample rate $T=1$.

(ii) Algorithms' Parameters. UKF controlled ACT model's parameter:

$$
\begin{gathered}
\alpha=10^{-3}, \quad \beta=2, \quad \kappa=0 \\
Q_{\mathrm{ACT} 1}=\operatorname{diag}\left\{\begin{array}{lll}
1 & 1 & 10^{-4}
\end{array}\right\} \\
Q_{\mathrm{ACT} 2}=\operatorname{diag}\left\{\begin{array}{ll}
1 & 10^{-4}
\end{array}\right\} .
\end{gathered}
$$

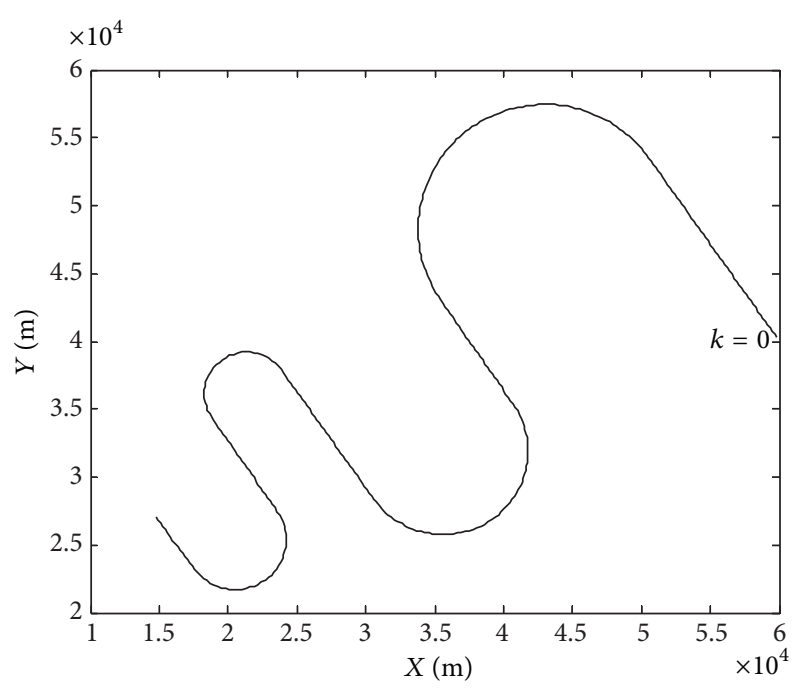

FIGURE 1: The test trajectory.

Kinematic constraint model's parameter:

$$
\begin{gathered}
Q_{\mathrm{CA}}=\operatorname{diag}\left\{\begin{array}{ll}
1 & 1
\end{array}\right\} \\
\delta=0.92, \quad r_{0}=1, \quad r_{1}=200 .
\end{gathered}
$$

Maneuver centered model's parameter:

$$
\begin{gathered}
Q_{m}=\operatorname{diag}\left\{\begin{array}{ll}
10^{6} & 10^{-4}
\end{array}\right\} \\
\rho=0.8 .
\end{gathered}
$$

(iii) Results. The four models are listed as follows.

Method 1: ACT model with Cartesian velocity.

Method 2: ACT model with polar velocity.

Method 3: kinematic constraint model.

Method 4: maneuver-centered CT model.

Root mean squared errors (RMSE) are used here for comparison. The RME position errors are defined as follows:

$$
\text { RMS.P.E }(k)=\sqrt{\frac{1}{M} \sum_{i=1}^{M}\left[\left(x_{k}^{i}-\hat{x}_{k}^{i}\right)^{2}+\left(y_{k}^{i}-\hat{y}_{k}^{i}\right)^{2}\right]},
$$

where $M=200$ are the Monte-Carlo simulation runs. $x_{k}^{i}$ and $y_{k}^{i}$ stand for the true position, while $\widehat{x}_{k \mid k}^{i}$ and $\hat{y}_{k}^{i}$ are the position estimates.

The RMS position errors of all but the first ten are shown in Figure 2.

Table 1 summarizes the average RMS of the position errors.

Table 2 summarizes the relative computational complexity, normalized to method 4 .

It can be seen from the figure and tables that method 2 has the best performance and its computational load is roughly 


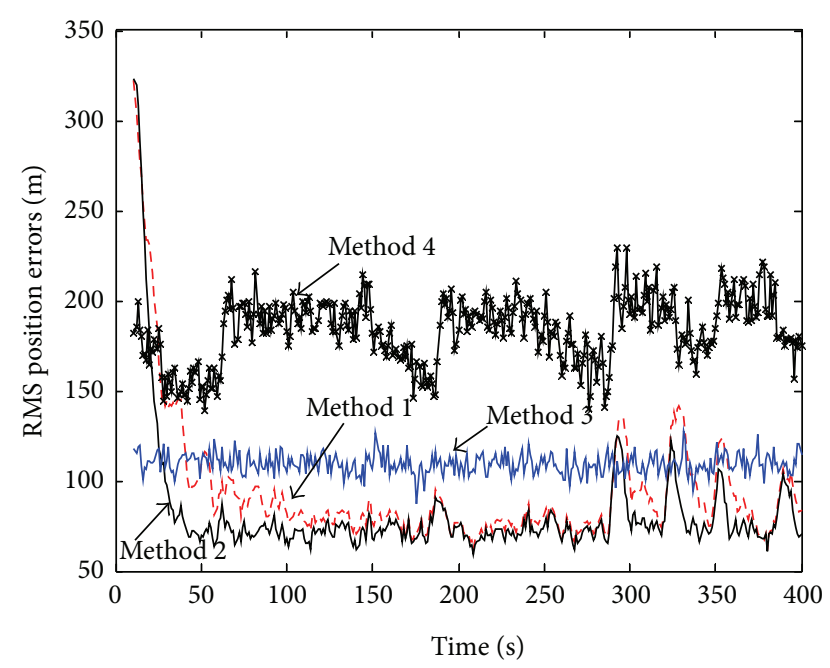

FIGURE 2: RMS position errors of the four methods.

TABLE 1: Average RMS of position errors.

\begin{tabular}{lc}
\hline Method & Average RMS of position errors (m) \\
\hline 1 & 94.26 \\
2 & 81.57 \\
3 & 109.51 \\
4 & 183.46 \\
\hline
\end{tabular}

TABLE 2: Relative computational load.

\begin{tabular}{lc}
\hline Method & Relative computational load \\
\hline 1 & 7.26 \\
2 & 7.07 \\
3 & 1.42 \\
4 & 1 \\
\hline
\end{tabular}

the same as method 1 . So we can conclude that ACT model with polar velocity is better than ACT model with Cartesian velocity. Method 4 has the least computational load but its performance is poor. Method 3 is slightly more complex than method 4 but can decrease the error greatly. So if the computational load is of great concern, kinematic constraint model is a good choice.

\section{The Expectation Maximization (EM) Algorithm for Tracking CT Motion Target}

In this part, the model in Section 2.1 is used.

The turn rate $\omega_{k}$ can be described by a Markov chain [21, 22] and has $r$ possible values:

$$
\omega_{k} \in M_{r}=\{\omega(1), \omega(2), \ldots, \omega(r)\} .
$$

Assume the initial probability $\tau_{i}$ and the one-step transition matrix are known, as follows:

$$
\begin{gathered}
\tau_{i}=p\left(\omega_{0}=\omega(i)\right), \quad i=1,2, \ldots, r \\
\pi_{i, j}=p\left(\omega_{k+1}=\omega(j) \mid \omega_{k}=\omega(i)\right), \quad i, j=1,2, \ldots, r .
\end{gathered}
$$

The measurement sequence is defined by $Z_{1: N}=$ $\left\{z_{1}, z_{2}, \ldots, z_{N}\right\}$, state sequence is $X_{1: N}=\left\{X_{1}, X_{2}, \ldots, X_{N}\right\}$, and maneuver sequence is $\Omega_{1: N}=\left\{\omega_{1}, \omega_{2}, \ldots, \omega_{N}\right\}$.

4.1. Batch EM Algorithm. Assume the measurement sequence is known, this algorithm focuses on finding the best maneuver sequence based on MAP criterion. There is one best maneuver sequence $\Omega_{1: N}^{(\mathbf{B})}$ in $r^{N}$ possible sequences that makes the conditional probability density function be the maximum. When $\Omega_{1: N}^{(\mathbf{B})}$ is achieved, the state sequence $X_{1: N}$ can be estimated accurately.

According to EM algorithm, $Z_{1: N}$ is considered to be the incomplete data, $X_{1: N}$ to be the "lost" data, and $\Omega_{1: N}$ to be the data that needs to be estimated. EM algorithm carries out the following two steps iteratively.

(1) Expectation Step (E step)

$$
\begin{aligned}
J & \left(\Omega_{1: N}, \Omega_{1: N}^{(j)}\right) \\
& =E_{\mathbf{X}_{1: N}}\left\{\ln p\left(X_{1: N}, Z_{1: N}, \Omega_{1: N}\right) \mid Z_{1: N}, \Omega_{1: N}^{(j)}\right\},
\end{aligned}
$$

where $J\left(\Omega_{1: N}, \Omega_{1: N}^{(j)}\right)$ is defined as the cost function, $\Omega_{1: N}^{(j)}$ is the maneuver sequence estimation after $j$ times iteration.

(2) Maximization step ( $M$ step)

$$
\Omega_{1: N}^{(j+1)}=\arg \max _{\Omega_{1: N}}\left(\Omega_{1: N}, \Omega_{1: N}^{(j)}\right) .
$$

If the initial value is given, the above E step and M step are carried out repeatedly, until convergence.

(i) E step. The union probability density function can be decomposed as follows:

$$
\begin{aligned}
p\left(X_{1: N}, Z_{1: N}, \Omega_{1: N}\right) \\
=\prod_{k=1}^{N} p\left(z_{k} \mid X_{k}\right) \times \prod_{k=1}^{N} p\left(X_{k} \mid X_{k-1}, \omega_{k-1}\right) \times p\left(X_{0}\right) \\
\quad \times \prod_{i=1}^{N} p\left(\omega_{i} \omega_{i-1}\right) \times p\left(\omega_{0}\right) .
\end{aligned}
$$

$p\left(X_{k} \mid X_{k-1}, \omega_{k-1}\right)$ and $p\left(\omega_{i} \mid \omega_{i-1}\right)$ rely on the maneuver sequence $\Omega_{1: N}$. The state equation is Gaussian distribution:

$$
p\left(X_{k} \mid X_{k-1}, \omega_{k-1}\right)=N\left\{X_{k}-F\left(\omega_{k-1}\right) X_{k-1}, Q_{k}\right\},
$$

where $N\{\mu ; \Sigma\}$ is the Gaussian probability density function with mean $\mu$ and covariance $\Sigma$. 
From the above analysis,

$$
\begin{aligned}
J\left(\Omega_{1: N}, \Omega_{1: N}^{(j)}\right) \\
=E_{X_{1: N}}\left\{\ln p\left(X_{1: N}, Z_{1: N}, \Omega_{1: N}\right) \mid Z_{1: N}, \Omega_{1: N}^{(j)}\right\} \\
=\sum_{k=1}^{N}\left\{\ln p\left(\omega_{k} \mid \omega_{k-1}\right)-\frac{1}{2}\left(\widehat{X}_{k \mid N}-F\left(\omega_{k-1}\right) \widehat{X}_{k-1 \mid N}\right)^{\prime}\right. \\
\left.\quad \times Q_{k}^{-1}\left(\widehat{X}_{k \mid N}-F\left(\omega_{k-1}\right) \widehat{X}_{k-1 \mid N}\right)\right\}
\end{aligned}
$$

where

$$
\widehat{X}_{k \mid N}=E\left[X_{k} \mid Z_{1: N}, \Omega_{1: N}^{(j)}\right]
$$
here.

Those terms which are independent of $\Omega_{1: N}$ are omitted

In the $\mathrm{E}$ step, if $\Omega_{1: N}^{(j)}$ is given, the cost function can be achieved using Kalman smoothing algorithm.

(ii) $M$ step. In the maximization step, a new $\Omega_{1: N}$ is chosen for a higher conditional probability. Then a better parameter estimation is achieved compared to the former iteration. The following Viterbi algorithm can solve this problem perfectly.

Viterbi algorithm is a recursive algorithm looking for the best path. As shown in Figure 3, the path connects the adjacent points with the weights to be the logarithm function of the likelihood, named cost. The path's total cost is the sum of its each point's cost. The best path has the maximum cost. The detailed method to find the best path can be found in [12].

\section{(iii) Calculating Algorithm}

(1) Initialization: the initial maneuver sequence $\Omega_{1: N}^{(1)}$ and threshold $\varepsilon$ should be given.

(2) Iteration: for each circle $(j=1,2, \ldots)$, carry out the following steps: (1) E step, according to (53), calculate the cost between the adjacent point. (2) M step, according to Viterbi algorithm, find a better maneuver sequence.

(3) Stop: if $\left\|\Omega_{1: N}^{(j+1)}-\Omega_{1: N}^{(j)}\right\| \leq \varepsilon$, then stop the iteration. The best maneuver sequence is $\Omega_{1: N}^{(\mathbf{B})}=\Omega_{1: N}^{(j+1)}$; then the state estimation sequence is calculated according to $\Omega_{1: N}^{(B)}$

4.2. Recursive EM Algorithm. In target tracking applications, the target's state always needs online estimation. So a recursive EM algorithm is needed for calculating $\omega_{k}$.

(i) Recursive Equation. Under the MAP criterion,

$$
\Omega_{1: k}^{(\mathbf{B})}=\arg \max _{\Omega_{1: k}}\left\{p\left(\Omega_{1: k} \mid Z_{1: k}\right)\right\},
$$

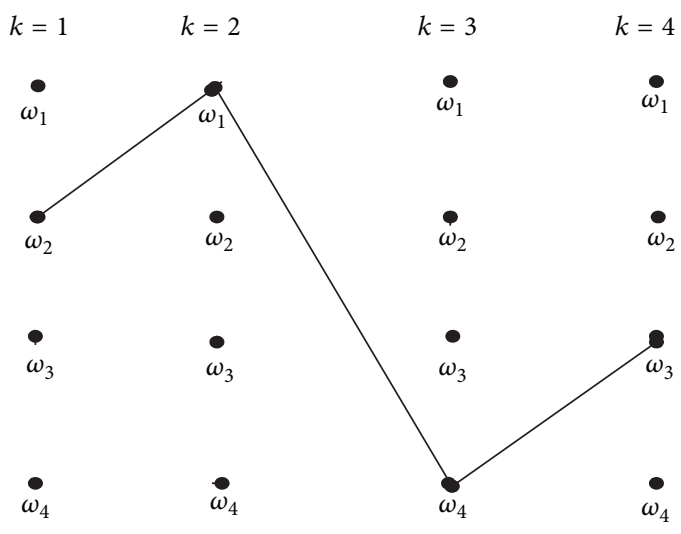

FIGURE 3: Viterbi algorithm for path following.

where $p\left(\Omega_{1: k} \mid Z_{1: k}\right)$ can be calculated online.

$$
\begin{aligned}
p\left(\Omega_{1: k} \mid Z_{1: k}\right)= & p\left(\Omega_{1: k} \mid z_{k}, Z_{1: k-1}\right) \\
= & \frac{p\left(z_{k} \mid \Omega_{k}, Z_{1: k-1}\right) p\left(\Omega_{k} \mid Z_{1: k-1}\right)}{p\left(z_{k} \mid \Omega_{1: k-1}\right)} \\
= & p\left(z_{k} \mid \Omega_{1: k}, Z_{1: k-1}\right) p\left(\omega_{k} \mid \Omega_{1: k-1}\right) \\
& \times p\left(\Omega_{1: k-1} \mid Z_{1: k-1}\right)\left(p\left(z_{k} \mid Z_{1: k-1}\right)\right)^{-1} .
\end{aligned}
$$

Because $\Omega_{1: k}$ is Markov chain,

$$
p\left(\omega_{k} \mid \Omega_{1: k-1}\right)=p\left(\omega_{k} \mid \omega_{k-1}\right) .
$$

The possible maneuver sequence grows exponentially as the time grows. For the computation to be feasibility, it is assumed that

$$
\begin{aligned}
p\left(z_{k} \mid \Omega_{1: k}, Z_{1: k-1}\right) & \approx p\left(z_{k} \mid \omega_{k}, Z_{1: k-1}\right) \\
& =N\left(\boldsymbol{v}_{k}, \mathbf{S}_{k}\right)
\end{aligned}
$$

where $\boldsymbol{v}_{k}$ is the Kalman filter's innovation and $\mathbf{S}_{k}$ is the covariance of the innovation.

The cost function is defined as

$$
J\left(\omega_{k}(i)\right)=\ln p\left(\Omega_{1: k}, \omega_{k}(i) Z_{1: k}\right), \quad i=1,2, \ldots, r
$$

which stands for the cost to model $i$ until time $k$.

From (57) to (59),

$$
\begin{aligned}
J\left(\omega_{k}(j)\right)= & J\left(\omega_{k-1}(i)\right)+\ln \pi_{i j} \\
& -\frac{1}{2} \boldsymbol{v}_{k}^{\prime}(i, j) \mathbf{S}_{k}^{-1}(i, j) \boldsymbol{v}_{k}(i, j) \quad i, j=1,2, \ldots, r
\end{aligned}
$$

where $\boldsymbol{v}_{k}(i, j)$ stands for the innovation when model $i$ is chosen in time $k-1$ and model $j$ is chosen in time $k . S_{k}(i, j)$ is the corresponding covariance. 
Because of using the assumption (58), the iteration algorithm is not the optimal algorithm under MAP criterion, but a suboptimal one.

(ii) Calculating Algorithm. Only one-step iteration is listed here.

(1) E Step Calculation. Using (10), calculate each cost from time $k-1$ to $k ; r^{2}$ costs are needed.

(2) M Step Calculation. According to Viterbi algorithm, find out the maximum cost $J_{\max }\left(\omega_{k}(i)\right)$ related to each model. $J_{\max }\left(\omega_{k}(i)\right)$ is the initial value to be the next iteration.

(3) Filtering. According to the path which reaches each model, calculate each model's state estimation $\widehat{X}_{k}(i)$ and covariance $P_{k \mid k}(i), i=1,2, \ldots, r$.

(4) The Final Results. From $J_{\max }\left(\omega_{k}(i)\right), i=1,2, \ldots, r$, choose the maximum one as the final filtering result:

$$
\begin{gathered}
j=\arg \max _{i}\left\{J_{\max }\left(\omega_{k}(i)\right)\right\}_{i=1}^{r} . \\
\widehat{X}_{k}^{(\mathbf{B})}=\widehat{X}_{k}(j), \quad P_{k \mid k}^{(\mathbf{B})}=P_{k \mid k}(j) .
\end{gathered}
$$

\subsection{Simulation Results}

(i) Simulation Scenario. Target initial state is $X_{0}=$ $[60000 \mathrm{~m}-172 \mathrm{~m} / \mathrm{s} 40000 \mathrm{~m} 246 \mathrm{~m} / \mathrm{s}]^{\prime}$. The sample rate $T=1 \mathrm{~s}$. The covariance of process noise

$$
Q=\left[\begin{array}{cc}
Q_{x} & 0 \\
0 & Q_{y}
\end{array}\right], \quad Q_{x}=Q_{y}=\left[\begin{array}{cc}
\frac{T^{4}}{3} & \frac{T^{3}}{2} \\
\frac{T^{3}}{2} & T^{2}
\end{array}\right]
$$

Assume only position can be measured, the measurement equation is the following:

$$
z_{k}=\left[\begin{array}{llll}
1 & 0 & 0 & 0 \\
0 & 0 & 1 & 0
\end{array}\right] X_{k}+v_{k}
$$

The covariance of measurement noise $R=2500 \mathrm{I}$, where I is the $2 \times 2$ unit matrix.

The simulation lasts for $300 \mathrm{~s}$. Target's true turn rate is

$$
\omega_{k}= \begin{cases}0 & 0 \leq k<103 \\ 0.033 \mathrm{rad} / \mathrm{s} & 104 \leq k<198 \\ 0 & 198 \leq k<300 .\end{cases}
$$

Figure 4 gives the target's true trajectory.

Assume target's maximum centripetal acceleration is $30 \mathrm{~m} / \mathrm{s}^{2}$. Under the speed $300 \mathrm{~m} / \mathrm{s}$, the corresponding turn rate is $0.1 \mathrm{rad} / \mathrm{s}$. Seven models are used for this simulation. From -0.1 to 0.1 , the seven models are distributed evenly. Their values are $-0.1,-0.067,-0.033,0,0.033,0.067$, and 0.1 . The initial probability matrix is

$$
\tau=\left[\begin{array}{lllllll}
\frac{1}{7} & \frac{1}{7} & \frac{1}{7} & \frac{1}{7} & \frac{1}{7} & \frac{1}{7} & \frac{1}{7}
\end{array}\right] .
$$

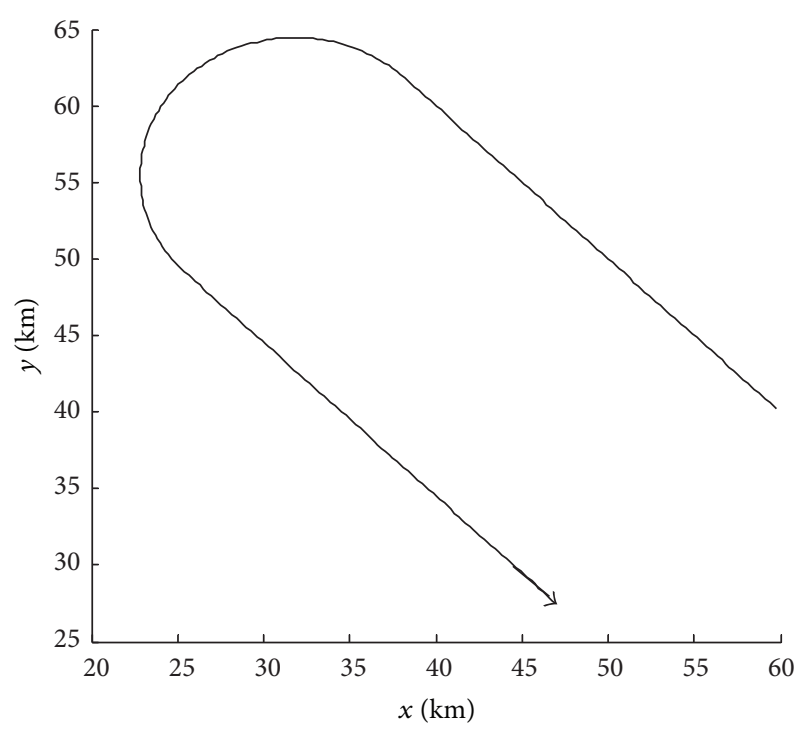

Figure 4: Target trajectory.

The model transition matrix is

$$
\begin{array}{r}
\pi_{i, j}= \begin{cases}0.7 & i=j \\
0.05 & i \neq j\end{cases} \\
i, j=1,2, \ldots, 7 .
\end{array}
$$

(ii) Simulation Results and Analysis. Batch EM algorithm, recursive algorithm, and IMM algorithm are compared in this scenario. Root mean squared errors (RMSE) are used here for comparison. The RME position errors are defined as (46) and velocity error are defined as follows:

$$
\text { RMS.V.E }(k)=\sqrt{\frac{1}{M} \sum_{i=1}^{M}\left[\left(\dot{x}_{k}^{i}-\hat{\dot{x}}_{k}^{i}\right)^{2}+\left(\dot{y}_{k}^{i}-\hat{\dot{y}}_{k}^{i}\right)^{2}\right]},
$$

where $M=200$ are Monte-Carlo simulation runs and $\dot{x}_{k}^{i}, \dot{y}_{k}^{i}$ and $\hat{\dot{x}}_{k}^{i}, \widehat{\dot{y}}_{k}^{i}$ stand for the true and estimated velocity at time $k$ in the $i$ th simulation runs, respectively.

Figures 5 and 6 show the position and velocity performance comparison. It can be concluded that the batch EM algorithm has much less tracking errors compared to IMM algorithm. During maneuver onset time and termination time, the IMM algorithm is better than recursive EM algorithm. But on stable period, the recursive EM algorithm performs better.

\section{Conclusions}

Aiming at the CT motion target tracking, several models and algorithms are introduced and simulated in this paper.

In single model framework, four CT models have been compared for tracking applications: ACT model with Cartesian velocity, ACT model with polar velocity, kinematic constraint model, and maneuver-centered model. The MonteCarlo simulations show that the ACT model with polar 


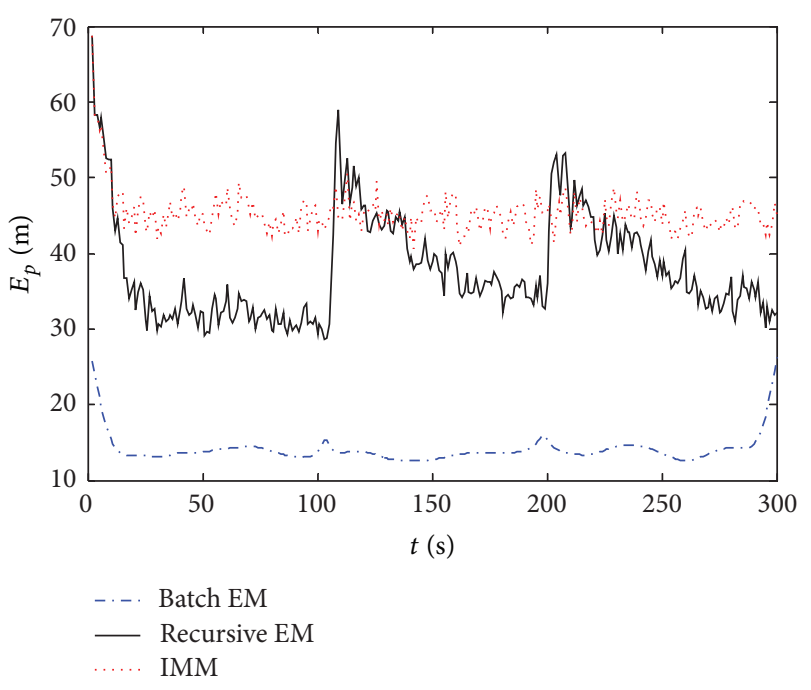

FIgURE 5: Position performance comparison.

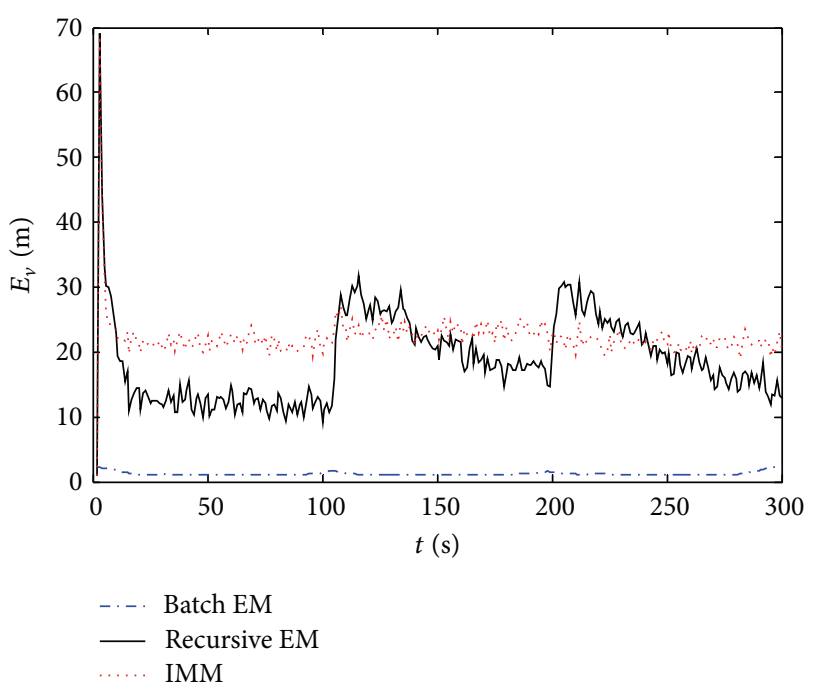

FIGURE 6: Velocity performance comparison.

velocity has the best tracking performance but the computational load is a bit heavier. The kinematic constraint model has a moderate tracking performance, but its computational load decreases greatly compared with UKF controlled ACT model. So if the computational load is of a great concern, the kinematic constraint model is suggested. If the tracking performance is very important and the computational load is not a problem, the ACT model with polar velocity is suitable.

In multiple models framework, EM algorithm is used for tracking CT motion target. First a batch EM algorithm is derived. The turn rate is acted as the maneuver sequence and estimated based on the MAP criterion. Under the E step, the cost function is calculated using the Kalman smoothing algorithm. Under the M step, Viterbi algorithm is used for path following to find out the path with maximum cost. Simulation results show that the Batch EM algorithm has better tracking performance than IMM algorithm. Through modification of the cost function, a recursive EM algorithm is presented. The algorithm can track the target online. Compared with the IMM algorithm, on the stable period, the recursive EM algorithm has better tracking performance.

\section{Conflict of Interests}

The authors declare that there is no conflict of interests regarding the publication of this paper.

\section{Acknowledgments}

This research work was supported by the National Key Fundamental Research \& Development Programs (973) of China (2013CB329405), Foundation for Innovative Research Groups of the National Natural Science Foundation of China (61221063), Natural Science Foundation of China (61203221, 61004087), Ph.D. Programs Foundation of Ministry of Education of China (20100201120036), China Postdoctoral Science Foundation (2011M501442, 20100481338), and Fundamental Research Funds for the Central University.

\section{References}

[1] X. R. Li and V. P. Jilkov, "Survey of maneuvering Target Tracking-part I: dynamic models," IEEE Transactions on Aerospace and Electronic Systems, vol. 39, no. 4, pp. 1333-1364, 2003.

[2] F. Gustafsson and A. J. Isaksson, "Best choice of coordinate system for tracking coordinated turns," in Proceedings of the 35th IEEE Conference on Decision and Control, pp. 3145-3150, Kobe, Japan, December 1996.

[3] A. J. Isaksson and F. Gustaffsson, "Comparison of some Kalman filter based on methods for maneuver tracking and detection," in Proceedings of the 34th IEEE Conference on Decision and Control, pp. 1525-1530, New Orleans, La, USA, December 1995.

[4] S. Julier, J. Uhlmann, and H. F. Durrant-Whyte, "A new method for the nonlinear transformation of means and covariances in filters and estimators," IEEE Transactions on Automatic Control, vol. 45, no. 3, pp. 477-482, 2000.

[5] S. J. Julier and J. K. Uhlmann, "Unscented filtering and nonlinear estimation," Proceedings of the IEEE, vol. 92, no. 3, pp. 401422, 2004.

[6] M. Tahk and J. L. Speyer, "Target Tracking problems subject to kinematic constraints," IEEE Transactions on Automatic Control, vol. 35, no. 3, pp. 324-326, 1990.

[7] A. T. Alouani and W. D. Blair, "Use of a kinematic constraint in tracking constant speed, maneuvering targets," in Proceedings of the 30th IEEE Conference on Decision and Control, pp. 10551058, Brighton, UK, December 1991.

[8] A. T. Alouani and W. D. Blair, "Use of a kinematic constraint in tracking constant speed, maneuvering targets," IEEE Transactions on Automatic Control, vol. 38, no. 7, pp. 1107-1111, 1993.

[9] J. A. Roecker and C. D. McGillem, "Target Tracking in maneuver-centered coordinates," IEEE Transactions on Aerospace and Electronic Systems, vol. 25, no. 6, pp. 836-843, 1989.

[10] X. R. Li and V. P. J. ILkov, "A Survey of maneuvering Target Tracking-part III: measurement models," in Conference on Signal and Data Processing of Small Targets, vol. 4437 of Proceeding of SPIE, pp. 423-446, San Diego, Calif, USA, 2001. 
[11] Y. Bar-Shalom, K. C. Chang, and H. A. P. Blom, "Tracking a maneuvering target using input estimation versus the interacting multiple model algorithm," IEEE Transactions on Aerospace and Electronic Systems, vol. 25, no. 2, pp. 296-300, 1989.

[12] A. Averbuch, S. Itzikowitz, and T. Kapon, "Radar Target Tracking-viterbi versus IMM," IEEE Transactions on Aerospace and Electronic Systems, vol. 27, no. 3, pp. 550-563, 1991.

[13] G. W. Pulford and B. F. La Scala, "MAP estimation of target manoeuvre sequence with the expectation-maximization algorithm," IEEE Transactions on Aerospace and Electronic Systems, vol. 38, no. 2, pp. 367-377, 2002.

[14] A. Logothetis, V. Krishnamurthy, and J. Holst, "A Bayesian EM algorithm for optimal tracking of a maneuvering target in clutter," Signal Processing, vol. 82, no. 3, pp. 473-490, 2002.

[15] P. Willett, Y. Ruan, and R. Streit, "PMHT: problems and some solutions," IEEE Transactions on Aerospace and Electronic Systems, vol. 38, no. 3, pp. 738-754, 2002.

[16] Z. Li, S. Chen, H. Leung, and É. Bossé, "Joint data association, registration, and fusion using EM-KF," IEEE Transactions on Aerospace and Electronic Systems, vol. 46, no. 2, pp. 496-507, 2010.

[17] D. L. Huang, H. Leung, and E. Boose, "A pseudo-measurement approach to simultaneous registration and track fusion," IEEE Transactions on Aerospace and Electronic Systems, vol. 48, no. 3, pp. 2315-2331, 2012.

[18] X. Yuan, C. Han, Z. Duan, and M. Lei, "Adaptive turn rate estimation using range rate measurements," IEEE Transactions on Aerospace and Electronic Systems, vol. 42, no. 4, pp. 1532-1540, 2006.

[19] V. B. Frencl and J. B. R. do Val, "Tracking with range rate measurements: turn rate estimation and particle filtering," in Proceedings of the IEEE Radar Conference, pp. 287-292, Atlanta, Ga, USA, May 2012.

[20] E. Semerdjiev, L. Mihaylova, and X. R. Li, "Variable- and fixedstructure augmented IMM algorithms using coordinated turn model," in Proceedings of the 3rd International Conference on Information Fusion, pp. 10-13, Paris, France, July 2000.

[21] Y. Bar-shalom, X. R. Li, and T. Kirubarajan, Estimation with Applications to Tracking and Navigation: Theory, Algorithms and Software, John Wiley \& Sons, New York, NY, USA, 2001.

[22] J. P. Iielferty, "Improved tracking of maneuvering targets: the use of turn-rate distributions for acceleration modeling," IEEE Transactions on Aerospace and Electronic Systems, vol. 32, no. 4, pp. 1355-1361, 1996. 


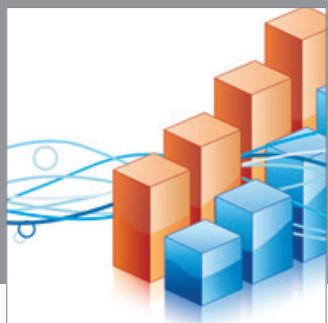

Advances in

Operations Research

mansans

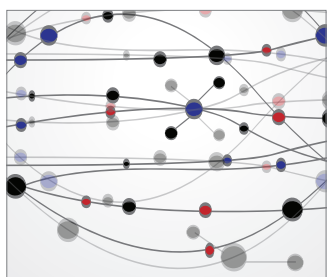

The Scientific World Journal
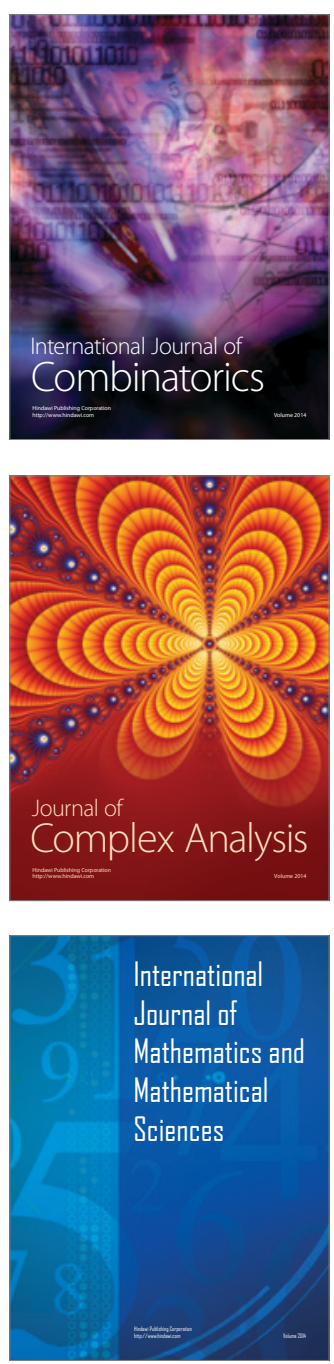
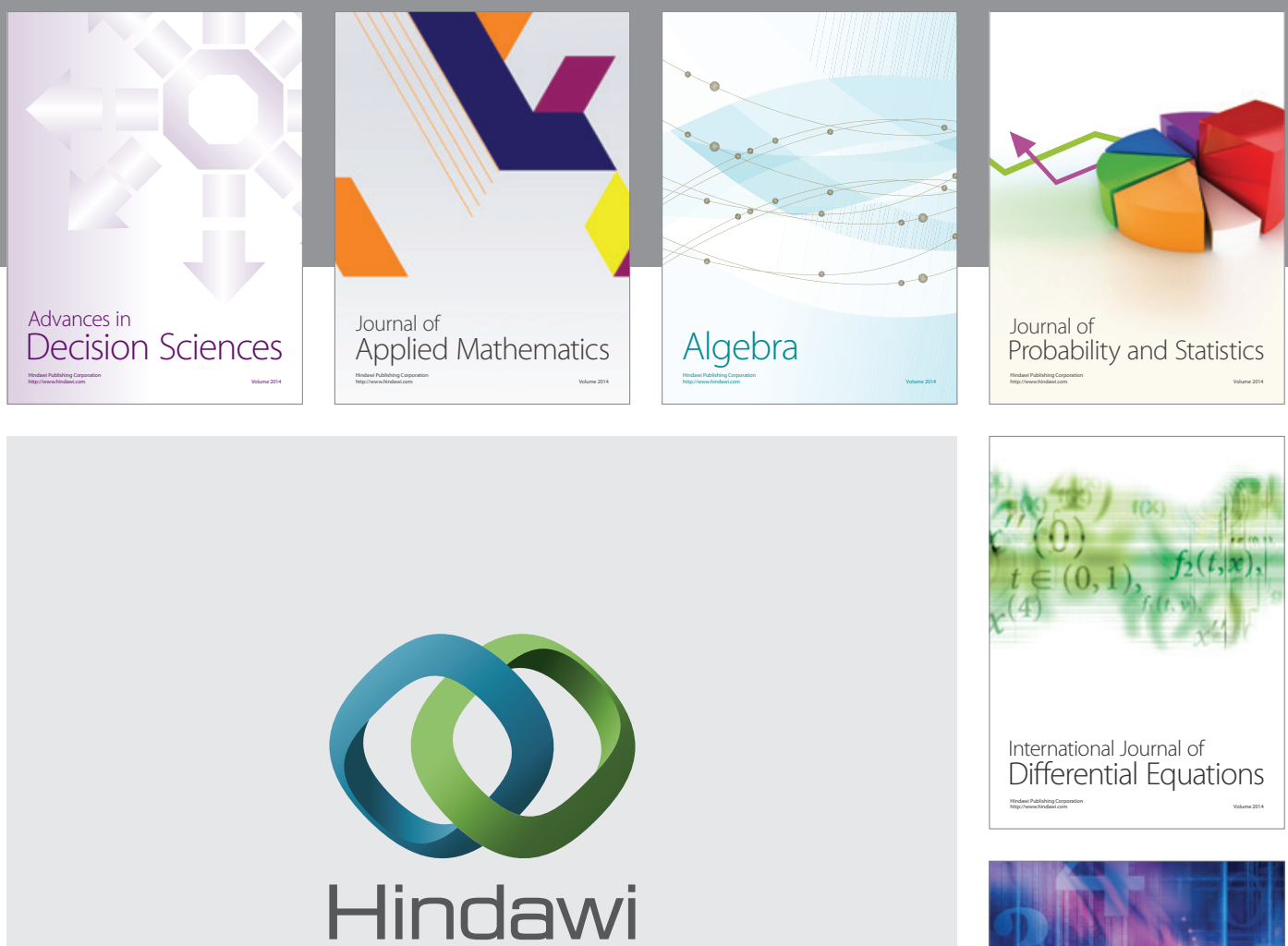

Submit your manuscripts at http://www.hindawi.com
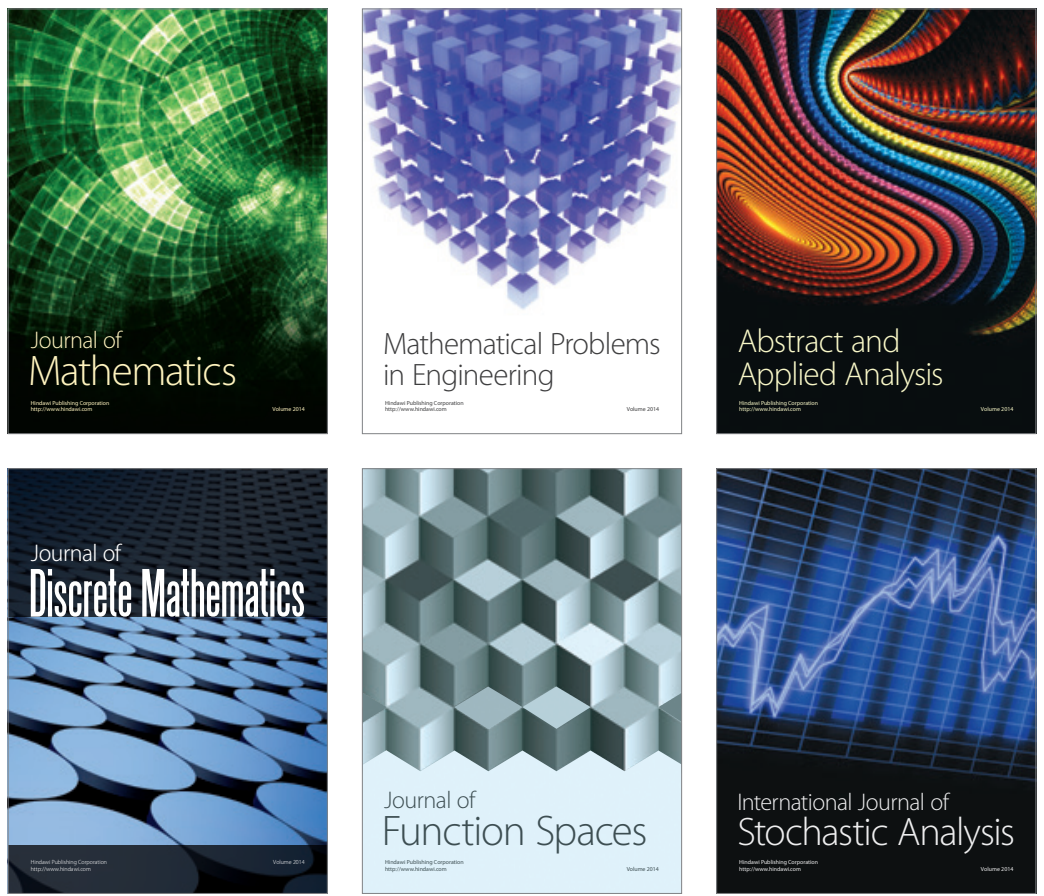

Journal of

Function Spaces

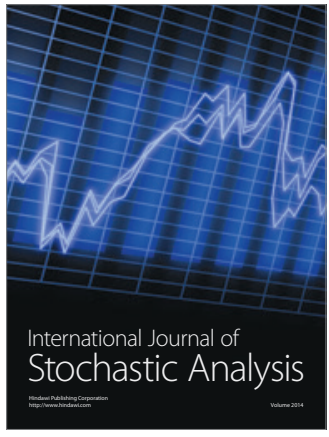

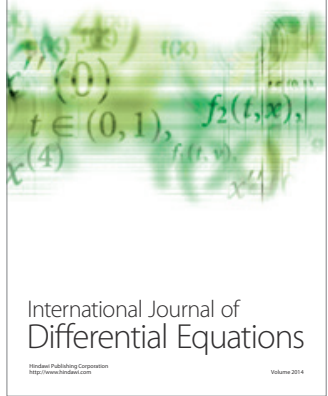
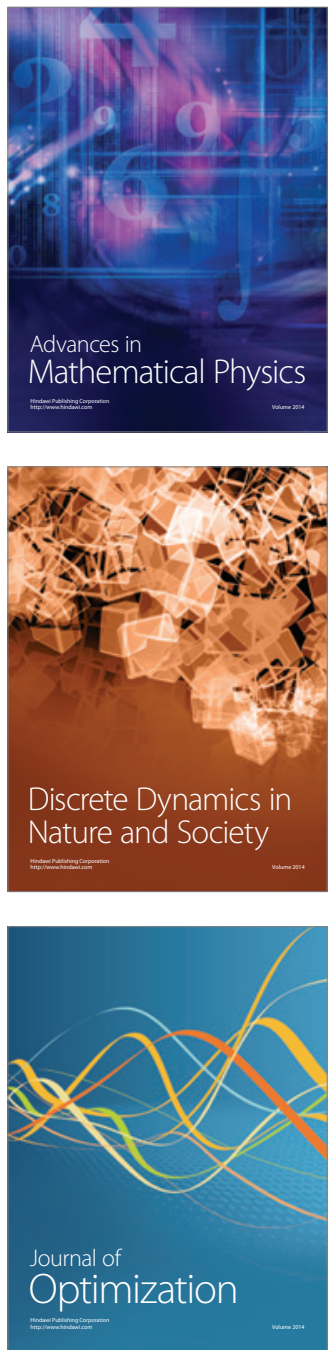